\title{
In vivo fiber photometry of neural activity in response to optogenetically manipulated inputs in freely moving mice
}

\author{
Liang $\mathrm{Li}^{*, \dagger}$, Yajie Tang ${ }^{*, \dagger}$, Leqiang Sun ${ }^{*, \dagger}$, Khaista Rahman*, ${ }^{*,}$ Kai Huang ${ }^{\boldsymbol{\top}, \|}$, Weize Xu ${ }^{*, \dagger}$, \\ Jinsong $\mathrm{Yu}^{*, \dagger}$, Jinxia Dai ${ }^{*, \dagger, \uparrow, * * * * *}$ and Gang Cao ${ }^{*, \dagger, \uparrow, \$, \dagger, * * *}$ \\ *State Key Laboratory of Agricultural Microbiology \\ Huazhong Agricultural University \\ Wuhan 430070, P. R. China \\ ${ }^{\dagger}$ College of Veterinary Medicine \\ Huazhong Agricultural University \\ Wuhan 430070, P. R. China \\ $\$$ Bio-Medical center \\ Huazhong Agricultural University \\ Wuhan 430070, P. R. China \\ ${ }^{\S}$ Key Laboratory of Development of Veterinary Diagnostic Products \\ Ministry of Agriculture, College of Veterinary Medicine \\ Huazhong Agricultural University, Wuhan 430070, P. R. China \\ "Wuhan National Laboratory for Optoelectronics \\ Huazhong University of Science and Technology \\ Wuhan, 430074, P. R. China \\ "Convergence Technology Co. Ltd \\ 1037 Luoyu Road, Hongshan District, Wuhan 430074, P. R. China \\ **jxdai@mail.hzau.edu.cn \\ †'gcao@mail.hzau.edu.cn
}

Received 12 February 2017

Accepted 15 March 2017

Published 21 April 2017

\begin{abstract}
In vivo fiber photometry is a powerful technique to analyze the dynamics of population neurons during functional study of neuroscience. Here, we introduced a detailed protocol for fiber photometry-based calcium recording in freely moving mice, covering from virus injection, fiber stub insertion, optogenetical stimulation to data procurement and analysis. Furthermore, we
\end{abstract}

\footnotetext{
${ }^{* * *}$ Corresponding authors.

This is an Open Access article published by World Scientific Publishing Company. It is distributed under the terms of the Creative Commons Attribution 4.0 (CC-BY) License. Further distribution of this work is permitted, provided the original work is properly cited.
} 
applied this protocol to explore neuronal activity of mice lateral-posterior (LP) thalamic nucleus in response to optogenetical stimulation of primary visual cortex (V1) neurons, and explore axon clusters activity of optogenetically evoked V1 neurons. Final confirmation of virus-based protein expression in V1 and precise fiber insertion indicated that the surgery procedure of this protocol is reliable for functional calcium recording. The scripts for data analysis and some tips in our protocol are provided in details. Together, this protocol is simple, low-cost, and effective for neuronal activity detection by fiber photometry, which will help neuroscience researchers to carry out functional and behavioral study in vivo.

Keywords: Fiber photometry; surgical operation; optogenetical stimulation; neural activity; freely moving recording.

\section{Introduction}

In vivo fiber photometry is a simple and costeffective system for optically recording real-time activity of neurons' dendrites, axons and soma body in freely moving animal combining with calcium ion $\left(\mathrm{Ca}^{2+}\right)$ indicators. ${ }^{1,2}$ The equipment is made up of the laser source, dichroscope, optical fiber coupler, fiber patch cable, fiber stub, optical filter, photomultiplier, amplifier, oscilloscope and computer. ${ }^{1,2}$ Fiber photometry detects the fluorescence change of $\mathrm{Ca}^{2+}$ concentration monitored by indicators such as BAPTA-AM, Fura2-AM, Fluo3-AM, Fluo4-AM, or genetically encoded calcium indicators (GECIs), etc. $^{3,4}$ The increase of $\mathrm{Ca}^{2+}$ concentration was generally used as a reporter of enhanced neuron activity due to $\mathrm{Ca}^{2+}$ influx into activated neuron. ${ }^{5,6}$ GCaMP protein is an extensively used GECI based on circularly permuted green fluorescent protein (cpGFP), calmodulin, ${ }^{7}$ and the $\mathrm{Ca}^{2+} / \mathrm{CaM}$-binding peptide (M13pep). ${ }^{6,8}$ The GCaMP indicator can be expressed in specific types of neurons driven by certain promoters,${ }^{9}$ even be localized to sub-cellular fraction by fusing with specific signal peptides. ${ }^{7}$ When binding with $\mathrm{Ca}^{2+}$, the fluorescent intensity of GCaMP rises from low to high, which can be detected by the photosensitive element. Currently, injection procedure of adeno-associated virus (AAV) with GCaMP gene into target site was elaborated and well described by Mathon et al. ${ }^{11}$ and Cetin et al. ${ }^{10}$ However, due to the lack of detailed operational protocol for fiber photometry, this technique is still difficult for beginner to handle during their study. While the in vivo fiber photometry of $\mathrm{Ca}^{2+}$ recording coupled with optogenetics technique is pivotal to explore neural function, the detailed step by step protocol is still scanty. Here, we introduced the detailed protocol of a simple, cost-effective method for in vivo fiber photometry coupled with optogenetics to detect neural activity. We applied GCaMP6s, a long potential duration and sensitive version of GCaMP calcium indicator, for calcium detection in freely moving mice. ${ }^{12}$ Based on GCaMP6s indicator and optogenetical stimulation, ${ }^{5,13,14}$ we recorded the real-time calcium waveform of lateral-posterior (LP) thalamic nucleus neurons in response to optogenetically stimulated V1 neurons in freely moving mice. Moreover, we also detected axonal calcium dynamics of optogenetically activated V1 neurons in target region LP by in vivo fiber photometry. Finally, the confirmation of virus injection and precise fiber insertion demonstrates that the procedure is reliable in operation accuracy for neuron activity recording. Our protocol will contribute to functional study of neural circuits and also help researcher quickly grasp and easily operate in vivo fiber photometry technique to their own study.

\section{Materials}

\section{Reagents}

- Experimental Mice (Two-month old Male C57BL/6 mice, use of live mice must conform to the Research Ethics Committee rules)

- Sterile Saline $(0.9 \% \mathrm{NaCl})$

- Anesthetics: Mixed anesthetics (7.5 g urethane (U2500-100 g, Sigma), $3 \mathrm{~g}$ chloral hydrate (Tianjin Damao Chemical Reagent Factory, China) and $75 \mathrm{mg}$ xylazine (X1251-5 g, Sigma) in $100 \mathrm{~mL} \mathrm{ddH}_{2} \mathrm{O}$ ); $1 \%$ Isoflurane (R510-22, RWD life science, China) in medical oxygen (Wuhan Shuanglonghe, China) 
- Dental Cement Kit (Super-Bond C\&B of Sun Medica, Japan): monomer, polymer (L-type Clear), catalyst V, spoon and ceramic disc (Fig. 1(a)).

- Viruses: AAV2/8-hSyn-hChR2(H134R)-mCherry in $1.13 \mathrm{E}+13$ V.G./mL titer (bought from Obio
Technology, China); AAV2/9-hSyn-GCaMP6s in $1.12 \mathrm{E}+13 \mathrm{~V} . \mathrm{G} . / \mathrm{mL}$ titer (bought from Obio Technology, China)

- Mineral oil and hot glue

— PBS and 4\% paraformaldehyde (PFA)
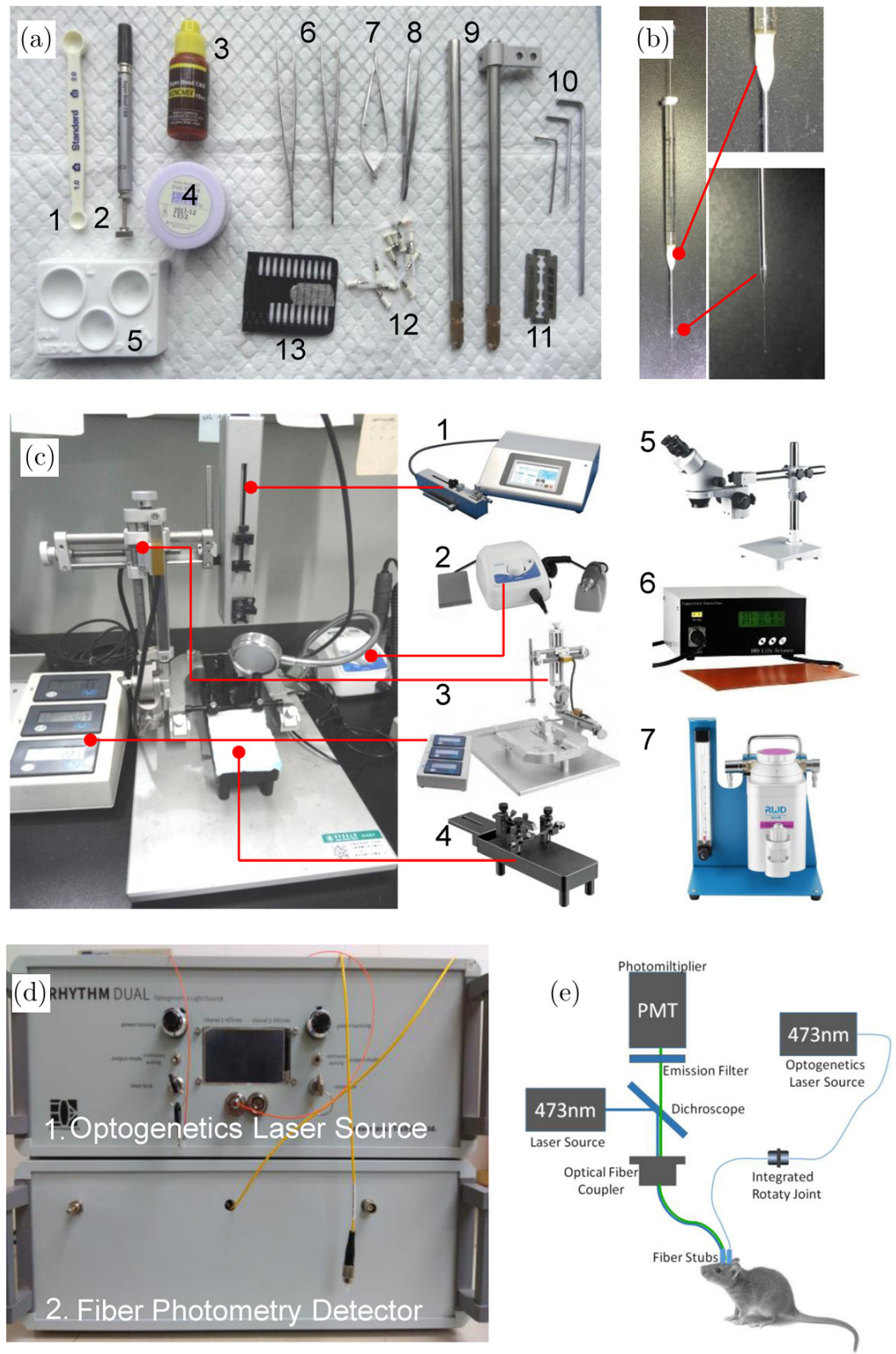

Notes: (a) and (b) Surgical tools and materials. A1, spoon for dental cement polymer; A2, catalyst V for dental cement; A3, monomer for dental cement; A4, L-type polymer; A5, ceramic disk for cement mixing; A6, fine forceps; A7, vanas scissors; A8, forceps; A9, fiber stub holders; A10, wrenches for holders; A11, blade for mouse hair shaving; A12, thin fiber stub for stimulation; A13, thick fiber stub for recording; B, virus microinjection needle made by pulled glass capillary fused with $10 \mu \mathrm{L}$ syringe by hot glue. (c) Equipments for microinjection. C1, KDS Legato ${ }^{\mathrm{TM}}$ 130 micro-pump; C2, high speed dental drill; C3, desktop digital stereotaxic instruments; C4, mouse adaptor; C5, stereomicroscope; C6, temperature controller; C7, small animal anesthesia machine (The images of C1-7 are from RWD website which were allowed for citation.). (d) Instruments for optogenetic stimulation and $\mathrm{Ca}^{2+}$ signal recording. D1, optogenetics laser source and manipulator; D2, fiber photometry detector. (e) Schematic diagram for in vivo $\mathrm{Ca}^{2+}$ signal recording by fiber photometry under laser stimulation.

Fig. 1. Materials and equipment for surgical operation of virus injection and fiber stub insertion. 


\section{Equipment}

- $10 \mu \mathrm{L}$ injection syringe (Tip diameter $0.7 \mathrm{~mm}$, Shanghai Gaoge industrial and trade, China) (Fig. 1(b))

- Borosilicate glass capillary with filament (O.D.: $1.5 \mathrm{~mm}$, I.D.: $0.86 \mathrm{~mm}$, \#BF150-86-10 from Sutter, USA)

- Hot glue gun

- Surgical tools: Vanas scissors (S11037-08, RWD life science, China), fine forceps (F11020-11, RWD life science, China), fiber stub holders (Convergence Technology, China), blade, sleeve (Convergence Technology, China), hexagon bar wrenches, thin fiber stubs $(\varnothing 62.5 \mu \mathrm{m}$, NA.0.22 for stimulation, Convergence Technology, China), thick fiber stubs (ø200 $\mu \mathrm{m}$, NA.0.37 for $\mathrm{Ca}^{2+}$ signal detection, Convergence Technology, China) (Fig. 1(a))

- Animal surgical pad (\#80098, RWD life science, China)

- Cotton swab

- Precision wipes (Kimtech science)

- $1 \mathrm{~mL}$ syringe with needle

- Stereomicroscope (\#77001, RWD life science, China) (Fig. 1(c))

- Mouse/Neonatal rat adaptor (\#68030, RWD life science, China) (Fig. 1(c))

- Desktop digital stereotaxic instruments (\#68025, RWD life science, China) (Fig. 1(c))

- Temperature controller (\#69001, RWD life science, China) (Fig. 1(c))

- High speed dental drill and $0.5 \mathrm{~mm}$ diameter drill-bits (\#78001 and \#78012, RWD life science, China) (Fig. 1(c))

- Puller PC-10 (DL Naturegene Life Sciences, USA)

- KDS Legato ${ }^{\mathrm{TM}} 130$ micro-pump (KD Scientific, USA) (Fig. 1(c))

- Rhythm dual optogenetics light source $473 \mathrm{~nm}$ and accessories (Convergence Technology, China) (Fig. 1(d))

- Fiber photometry from Convergence Technology (Emission Filters, bandpass 500-550 nm, Dichroscope, $491 \mathrm{~nm}$, Semrock; Photomultiplier, Hamamatsu Photonics; Laser source; Photocoupler; Oscilloscope, DSView) (Fig. 1(d))

- Small animal anesthesia machine-Isoflurane (R510iIP, RWD life science, China) (Fig. 1(c))

- Oxygen pressure relief (R-PG, RWD life science, China)
- Small animal video tracking system: Color video camera (Sony), HW-SET DVC100 Rev. 1.1 (Dazzle), Lens (1/3 CCTV CS, IR, $2.8 \mathrm{~mm}$, $1: 1.2$, Tamron)

- Software: AMCap9.11 for camera, DSView v0.9.6, Image J, Python for recorded data filtration, Matlab 2012b, Notepad ++

\section{Procedures:}

(1) Stereotaxic microinjection of virus in adult mice brain

\section{Anesthesia}

Mice were anesthetized by intraperitoneal (i.p.) injection of mixed anesthetics at $900 \mu \mathrm{L}$ per $100 \mathrm{~g}$ body weight. Then mice were fastened in the stereotaxic apparatus after squeezing the hind limb to ensure its deep anesthesia (Fig. 2(a)). Shaved the fur on the mouse scalp and removed head skin by scissors to expose skull for dental cement adhesion. Apply lubricant ophthalmic gel to both eyes to avoid keratitis during surgery.

\section{Mouse skull horizontal calibration}

For successful virus injection and fiber stub embedding, we need to do the calibration to ensure the top of mice skull is in the horizontal plane. Bregma point is set as the origin of anterior to posterior (AP), medial to lateral (ML) and dorsal to ventral (DV). From bregma to lamda, the distance is $4.10 \mathrm{~mm}$ to $4.40 \mathrm{~mm}$ depending on mice age 7 to 10 weeks,${ }^{14}$ so the location of lamda point would be AP:-4.10--4.40, ML: 0.00, DV: \pm 0.03 (Fig. 2(b)). Besides the bregma-lamda axis, the alignment of medial to lateral axis is necessary. We chose the point (AP:-2.00, ML: 0.00, DV: Z0, Z0 is the value on the skull) as middle and marked it. The left hemisphere point (AP:-2.00, ML: 2.50, DV: Z1) and the right hemisphere point (AP:-2.00, ML: $-2.50, \mathrm{DV}$ : Z2) should be adjusted to the same altitude $(\mathrm{Z} 1=\mathrm{Z2})$. $\mathrm{A} \pm 0.03 \mathrm{~mm} \mathrm{Z}$ value shift is acceptable in skull horizontal calibration (Fig. 2(b)). Usually, it takes $5 \mathrm{~min}$ to do the calibration.

\section{Virus injection}

Locate the injection site (Fig. 2(c)) and drill a hole gently by $0.5 \mathrm{~mm}$ diameter to expose the dura (Fig. 2(d)). Cover the hole by sterile saline to keep it moist, and then fix the injector (made by fusing $10 \mu \mathrm{L}$ syringe with electrode through hot glue and 

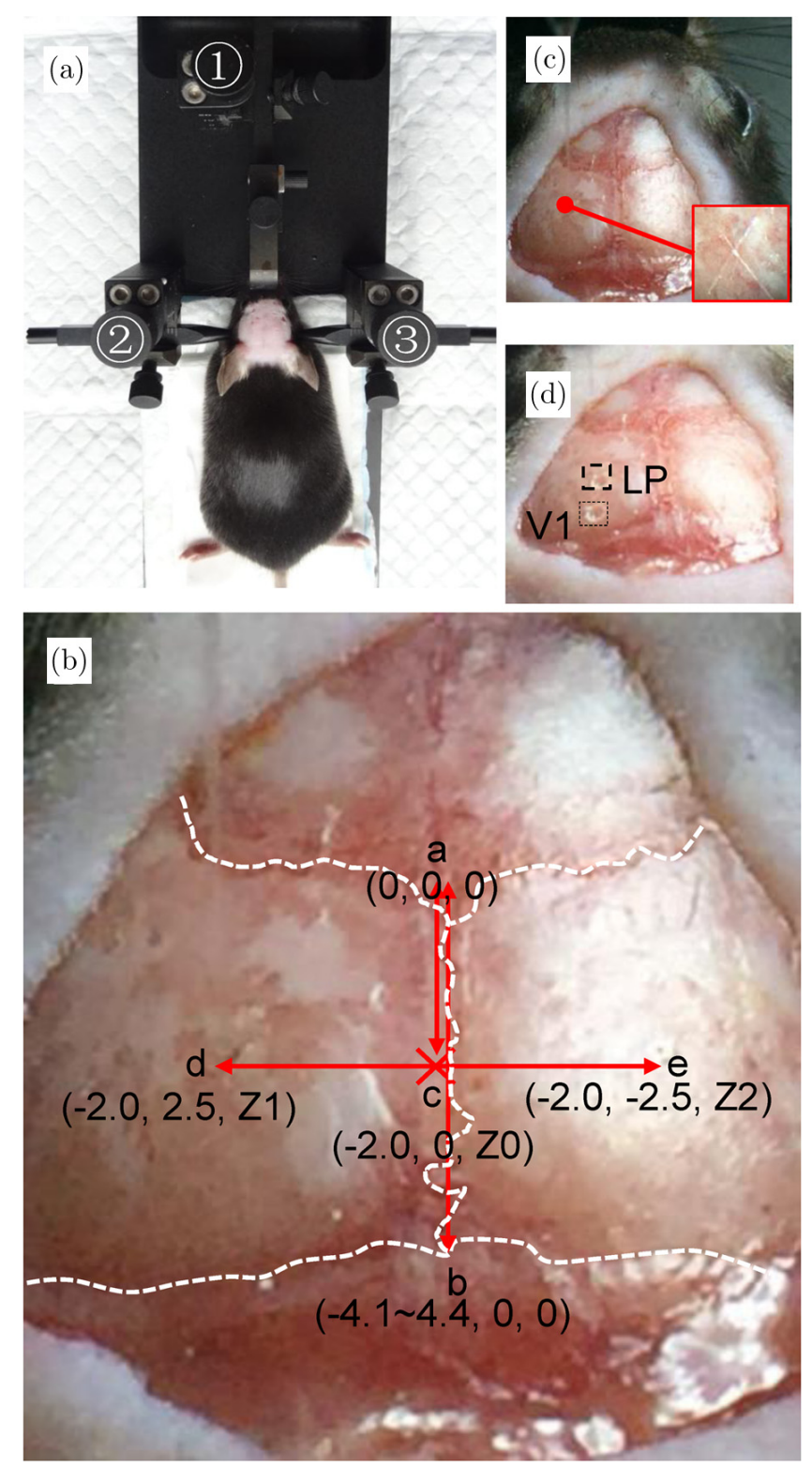

Notes: (a) Anesthetized mouse is fastened on adaptor after shaving. (1), screws for adjusting the incisor adaptor to calibrate horizontal plane along bregma-lamda axis; (2) and (3), screws for adjusting level of two ear bars to calibrate horizontal plane along medial-lateral axis. (b) Horizontal plane calibration strategy. a, coordinates of bregma; b, coordinates of lamda, the attitude of a and b ( $\mathrm{Z}$ value) should be same; $\mathrm{c}$, coordinates of reference point; $d$ and $e$, the symmetry point coordinates of head skull. When $\mathrm{Z} 1=\mathrm{Z} 2$, the medial-lateral axis is in horizontal plane. (c) Determination of target sites $\mathrm{X}$ and $\mathrm{Y}$ coordinates. The enlarged image shows the target site labeled by small cross with blade. (d) Burr-hole on skull for target sites (V1 and LP) and opening of the dura.

Fig. 2. Horizontal calibration of mouse skull during stereotaxic surgery.

filled it with mineral oil) on the micro-drive arm of micro pump (KDS Legato ${ }^{\mathrm{TM}} 130$ micro-pump). The virus was loaded into injector through direct sucking or sucking by micro pump. Totally $300 \mathrm{~nL}$ of
AAV2/8-hSyn-ChR2-mCherry virus were injected into $\mathrm{V} 1$ region (AP: -3.80 , ML: 2.50, DV: 0.40, $0.65)$ or AAV2/9-hSyn-GCaMP6s virus into LP nucleus (AP: -2.22 , ML: 1.60, DV: 2.62 ) at $20 \mathrm{~nL} /$ min for $15 \mathrm{~min}$ (Figs. 3(a) and 3(b)). The saline on skull surface should be cleaned before loading injector to target site and added again after the injector loading. Additional $5 \mathrm{~min}$ of rest time before withdrawing the injector is needed to avoid virus backflow. Totally, we take $45 \mathrm{~min}$ to finish microinjection of both AAV2/8-hSyn-ChR2-mCherry and AAV2/9-hSyn-GCaMP6s virus to each mouse.

\section{(2) Fiber stub embedding}

Remove saline and dry the skull with wipes and cotton swabs. Replace the micro-drive arm of micro pump with stub holder (Fig. 3(c)) and penetrate thick fiber stub with $2.62 \mathrm{~mm}$ depth from dura into LP (AP: -2.22 , ML: 1.60, DV:2.62), which was used for $\mathrm{Ca}^{2+}$ signal detection of LP nucleus. Dental cement was made up in pre-cold ceramic disc with 4 drop monomer, 1 drop catalyst and 1 small flat spoon polymer. A tiny of Tack-It clay was put on the fiber penetration site at V1 to avoid hole clogged by the cement (Fig. 3(d)). Subsequently, the whole skull was covered by the dental cement in multi-layers to strengthen the fiber stub connection with skull. Wait about $5 \mathrm{~min}$ for cement being solidified and carefully remove Tack-It clay by fine scissors. Additional $5 \mathrm{~min}$ later, fiber stub tightly fastened on skull can be released from holder. Another thin fiber stub for optogenetical stimulation should be embedded in V1 (AP: -3.80, ML: 2.50, DV: 0.40$)$ with $0.40 \mathrm{~mm}$ depth from dura at an angle of $30^{\circ}$ (the angle is on the AP vertical plane), since horizontal distance between V1 stimulation site and LP nucleus is too close to afford both stubs of optogenetic fiber and photometry fiber (Fig. 3 (e)). The same procedure was performed to make cement as the thin fiber stub fastening (Figs. 3(f) and $3(\mathrm{~g}))$. After dual fiber stub embedding, mouse was transferred onto $37^{\circ} \mathrm{C}$ temperature controller till to wake up. The surgical operation of dual fiber stub insertion needs about $50 \mathrm{~min}$ totally depending on personal experience, which contains $\sim 20$ min of dental cement solidification for each fiber.

\section{(3) $\mathbf{C a}^{2+}$ signal detection}

The surgical mice should be cared for $2-3$ weeks to ensure the target proteins expression of AAV virus 


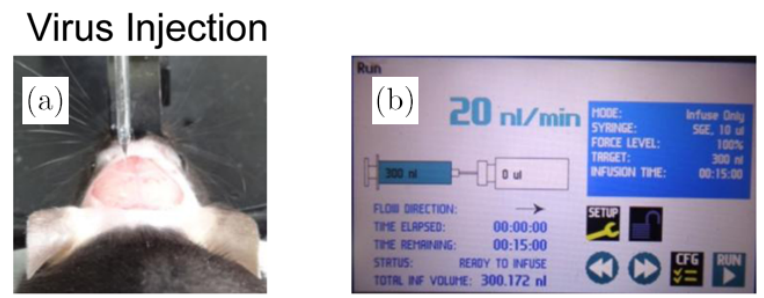

Fiber Stubs Insertion
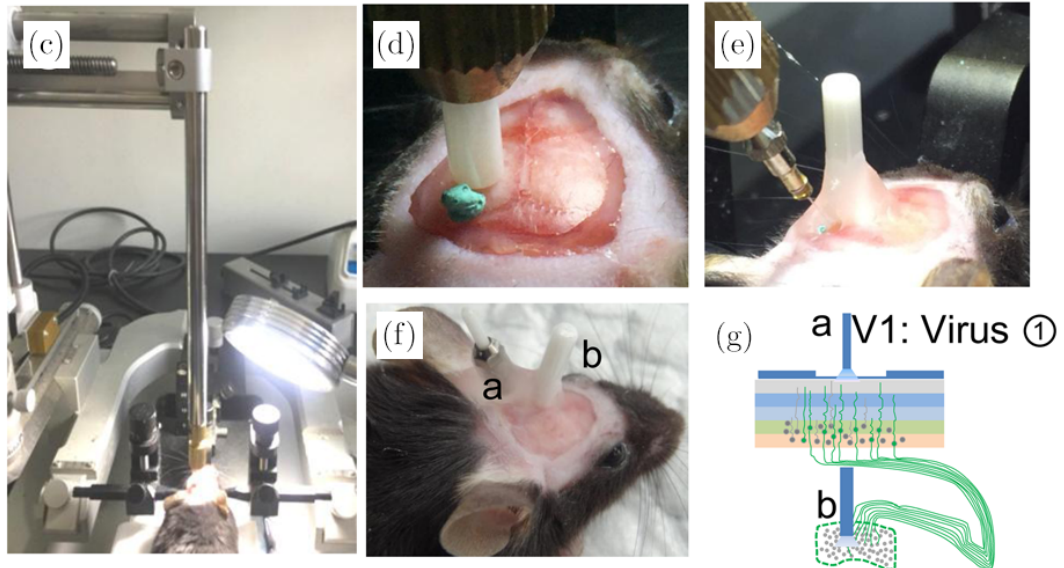

LP: Virus (2)
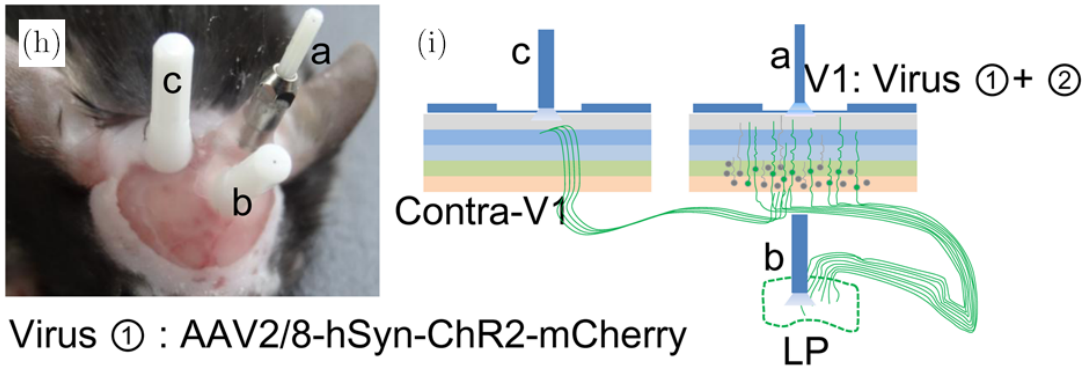

Virus (1) : AAV2/8-hSyn-ChR2-mCherry

Virus (2) : AAV2/9-hSyn-GCaMP6s

Notes: (a) Inserting needle to the target LP coordinate and injecting virus. (b) The injecting speed is $20 \mathrm{~nL} / \mathrm{min}$ and total volume of injected virus is $300 \mathrm{~nL}$. (c) Replacing the micro-drive for injector by fiber stub holder. (d) Loading the thick fiber stub (for calcium recording) to target coordinates and covering the other burr-hole by Tack-It clay. (e) Inserting the thin fiber stub (for optogenetical stimulation) to the V1 coordinates at $30^{\circ}$ angle of skull horizontal plane along anterior-posterior axis after recording stub is fixed by dental cement. (f) Accomplished insertion of stimulation fiber stub in V1 (a) and recording fiber stub in LP (b) for detecting LP neuron calcium dynamics. (g) Diagram of optogenetical stimulation in V1 and fiber photometry recording of LP neurons. "a" is thin fiber stub for optogenetical stimulation, "b" is thick fiber stub for Ca2 $2^{+}$signal recording; V1 was injected with AAV2/8-hSyn-ChR2-mCherry (virus (1), LP was injected with AAV2/9-hSyn-GCaMP6s (virus (2)). (h) Accomplished insertion of stimulation fiber stub in V1 (a) and recording fiber stub in LP (b) and contralateral V1 (c) for axonal calcium detection of V1 neurons. (i) Diagram of optogenetical stimulation in V1 and calcium activity recording of stimulated axon terminals targeting on LP and contralateral V1 (Contra-V1) after V1 neurons are activated. "a" is stimulation fiber stub, "b" and "c" are recording fiber stubs for axons in LP and contra-V1, respectively. Both AAV2/8-hSyn-ChR2-mCherry and AAV2/9-hSyn-GCaMP6s viruses (1) and (2)) are injected to V1.

Fig. 3. Virus microinjection and fiber stub insertion.

before $\mathrm{Ca}^{2+}$ signal recording. The instruments for optogenetical stimulation and fiber photometry were customized. Mice were anesthetized in animal anesthesia machine with isoflurane (isoflurane at $1 \%$ in oxygen, oxygen flow at $200 \mathrm{~mL} / \mathrm{min}$ ). The peak output stimulation of optogenetic fiber is $700 \mu \mathrm{W}$ at $100 \mathrm{~ms}$ duration and $5 \%$ duty ratio for 100 stimuli. And fiber photometry output is recorded by oscilloscope of DSCope (DreamSource Lab) and analyzed by DSView v0.9.6 software (as shown in Fig. 4(a) and supplemental video). The sampling rate is $20 \mathrm{kHz}$ and the signal amplifier for photomultiplier (PMT) is $10^{6}$ times. In freely moving mice, $\mathrm{Ca}^{2+}$ signal of thalamic LP nucleus was recorded (Fig. 4). Another camera (HW-SET DVC100 Rev. 1.1, Sony) was used for monitoring 

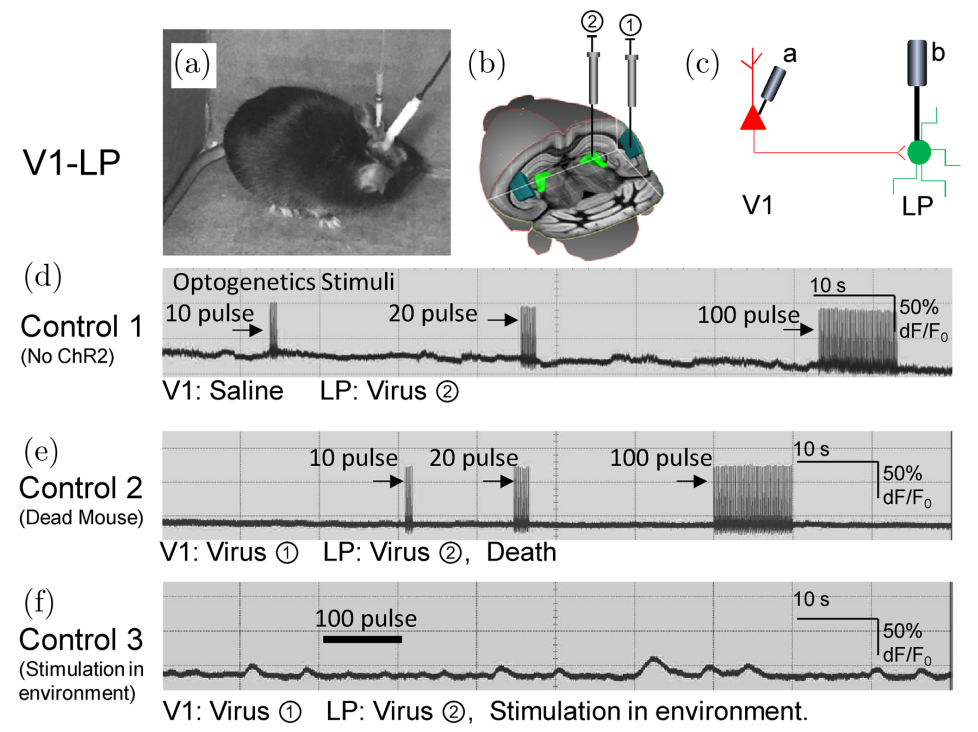

(g)

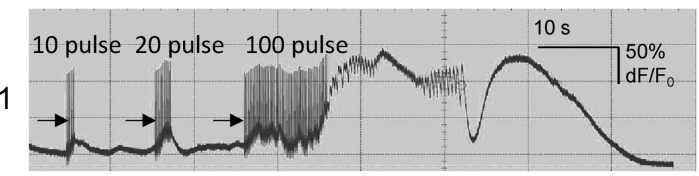

V1: Virus (1) LP: Virus (2)

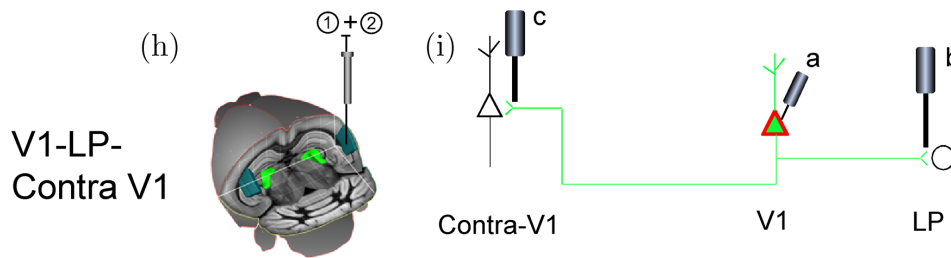

(j)

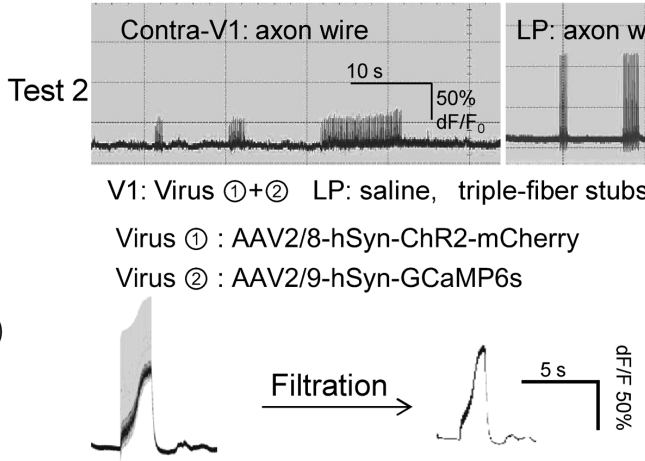

Notes: (a) Fiber photometry of LP neurons in freely moving mouse received laser stimulation in V1. (b, c) Diagram of virus injection and fiber insertion for V1-LP Ca ${ }^{2+}$ activity pathway detection. (d) Control 1 is fiber photometry data recorded in LP neuron of mouse without AAV2/8-hSynChR2-mCherry injection (1) in V1, but only with AAV2/9-hSyn-GCaMP6s (2)) virus injection in LP. No response was observed in LP neuron under 10, 20 and 100 pulses laser stimulation in V1. The black arrows indicate signal artifact of 10, 20 and 100 pulses optogenetical stimulation, which should be filtered by arithmetic. (e) Control 2 is fiber photometry data acquired in dead mouse with AAV2/8-hSyn-ChR2-mCherry (1) injection in V1 and AAV2/9-hSyn-GCaMP6s (2) injection in LP. No response but optogenetical stimulation artifact was detected in LP neuron under 10, 20 and 100 pulses laser stimulation in V1 as indicated by black arrows. (f) Control 3 is the recording data in LP neuron of virus (1) and (2) injected mouse when fiber cable stimulation is set in environment for 100 pulse. Black bar indicates laser stimulation. No response was detected in LP neuron under laser stimulation in environment. (g) Test 1 is the recording data of LP neuron in freely moving mouse with injected both virus (1) and (2) under 10, 20 and 100 pulses, respectively. The increase of LP neuron calcium dynamics can be detected under laser stimulation in V1. The black arrows indicate signal artifact of optogenetical stimulation at 10, 20 and 100 pulse. (h, i) The sketch of virus injection and fiber stub insertion for calcium recording of V1 axons projecting to LP and contralateral V1 (Contra-V1). (j) Axonal $\mathrm{Ca}^{2+}$ signal of activated V1 neurons targeting on Contra-V1 (left panel) and LP (left panel). (k) Typical $\mathrm{Ca}^{2+}$ signal after filtering the noise of optogenetical stimulation artifact and instruments generated high frequency wave.

Fig. 4. Fiber photometry of LP neurons and V1 neuronal axons $\mathrm{Ca}^{2+}$ dynamics in response to optogenetical stimulation of V1 in freely moving mice. 
the mice behavior during laser stimulation $(473 \mathrm{~nm})$ and $\mathrm{Ca}^{2+}$ signal recording (supplemental video).

\section{(4) Perfusion and Brain Dissection}

After fiber photometry, mice were anesthetized by mixed anesthetics (i.p.) and transcardially perfused by $4 \%$ PFA in $1 \times$ PBS. It is necessary to confirm the accuracy of virus injection and correct site of fiber stub insertion according to the fluorescence protein expression in injection area and position of stubs' well (Figs. 5(a) and 5(b)) respectively. Mice brains were dissected out and sectioned by vibratome (Leica VT1200S), then brain slices were imaged by auto-staged fluorescent microscope (Olympus, BX63). The whole process of animal perfusion, brain dissection, sectioning and slice
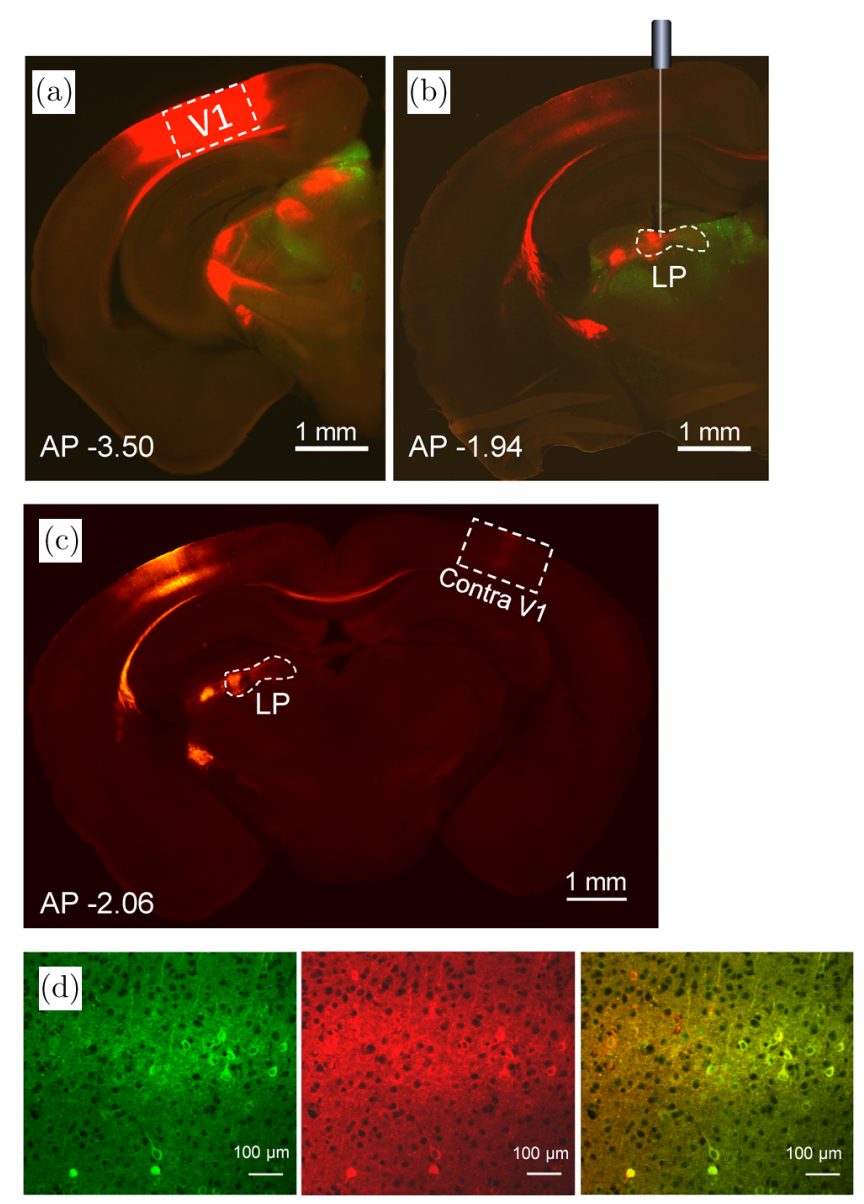

Notes: (a) AAV2/8-hSyn-ChR2-mCherry virus was accurately injected into V1 and expressed mCherry after two weeks. (b) AAV2/9-hSynGCaMP6s virus expressing GcamP6s was accurately injected into LP, and recording fiber was inserted into LP. (c) Clear projection of virus injected V1 neurons to LP and contralateral V1. (d) Injection site of virus (1) and (2) mix in V1 showing the co-expression of ChR2-mCherry and GCaMP6s.

Fig. 5. Confirmation of virus injection and fiber insertion. imaging can be finished in $24 \mathrm{~h}$. The imaging for each brain slice by fluorescent microscope takes 3 min.

\section{(5) Data Filtration}

During fiber photometry, the real calcium signal exported from oscilloscope was mixed with high frequency noise and optogenetical stimulation artifact, which should be filtered by two custom scripts based on python software. The supplemental text 1 is a script for $100 \mathrm{~Hz}$ low-pass filtration to remove high frequency noise caused by fiber photometry instruments. Then the filtered data was further processed by the other script (shown in supplemental text 2) to remove the optogenetical stimulation artifact. The final filtered data would be transformed into graphical plot of $\left(\mathrm{F}-\mathrm{F}_{0}\right) / \mathrm{F}_{0}$ ratio by Matlab software (Fig. 4(k)).

\section{Results}

We recorded $\mathrm{Ca}^{2+}$ dynamics of LP thalamic nucleus neurons and V1 neurons' axons targeted in LP nucleus and contralateral V1 (contra-V1) by fiber photometry combining with optogenetical stimulation in freely moving mice. When mice were injected with AAV2/9-hSyn-GCaMP6s in LP but not AAV2/8-hSyn-ChR2-mCherry injection in V1 (control 1 in Fig. 4), only stimulation artifact but not calcium response was detected in LP neurons under laser stimulation in V1 (Fig. 4(d)). Similarly, no calcium response was observed in LP neurons of dead mice, although mice were injected with AAV2/9-hSyn-GCaMP6s in LP and AAV2/8hSyn-ChR2-mCherry injection in V1 alive (control 2 in Fig. 4(e)). When laser stimulation was set in the environment instead of mouse V1, no response even stimulation artifact were detected in LP neurons with virus injection (control 3 in Fig. 4(f)), ruling out the possibility of artifact interference from retina by environment light. Under laser stimulation of 10, 20 and 100 pulses in V1, calcium waves were recorded in LP neurons of virus injected mice (test 1 in Fig. 4(g)), indicating LP neurons respond to activation of V1 neurons with ChR2 expression. Postmortem sectioning and imaging confirmed the accuracy of virus infection (Fig. 5(a)) and the recording fiber insertion (Fig. 5(b)). Furthermore, calcium response was also detected in axon cluster of ChR2 expressed V1 neurons in 
target region LP under laser stimulation in soma of V1 neurons (shown in Fig. 4(j)), suggesting this fiber photometry protocol is also applied to axon cluster's $\mathrm{Ca}^{2+}$ detection. The neural activity of V1 neurons' axons in LP can be recorded, although its amplitude is lower than that of soma $\mathrm{Ca}^{2+}$ signal of LP neurons (Fig. 4(j)). However, there is hardly any calcium response in V1 neurons' axons targeting on contra-V1 under laser stimulation (Fig. 4(j)), which may be due to lower density of $\mathrm{V} 1$ projection in contra-V1 than that in LP (Fig. 5(c)). The co-infection of AAV2/9-hSyn-GCaMP6s and AAV2/8hSyn-ChR2-mCherry virus in V1 lead to the coexpression of ChR2 and GCaMP6s protein in V1 neurons (Fig. 5(d)), although the fluorescent intensity of GCaMP6s is low. Taken together, these results showed that this fiber photometry protocol is reliable to record the soma and axon $\mathrm{Ca}^{2+}$ activity of neurons.

\section{Discussion}

\subsection{Identification of Bregma and coordinate}

Determination of accurate coordinate for virus injection to specific brain nucleus is a primary key step during microinjection operation, which largely depends on correct verification of bregma and lamda points. When opening head skin, sagittal suture is clear under moist skull, then suture can be labeled by fine forceps. The intersection of superior coronal sinus and sagittal suture is bregma point. After referring to the coordinate of specific target site from The Mouse Brain in Stereotaxic Coordinates (George Paxinos and Keith B. J. Franklin, Second Edition), it is necessary to adjust the coordinates by practicing injection of dye (Fluorescence Dye: Carboxyfluorescein diacetate, succinimidyl ester or Evans Blue) followed by immediate checking of brain section to finally verify its accurate coordinates, as a result of the coordinate shifting a little within mice in different laboratories.

\subsection{Fiber stub}

The thick fiber stub for $\mathrm{Ca}^{2+}$ signal recording is customized of the particular length and polished flat end which is unchangeable. The thin fiber stub for optogenetical stimulation can be truncated by optical fiber cutter, and polished by fire quickly.

\subsection{Anesthetic during surgical operation}

The surgical duration would be beyond of anesthetic efficacy, so it is necessary to supply anesthetic during surgery. When mouse beard shake fast, it is the time to add extra anesthetic. The additional anesthetic is $0.05 \mathrm{~mL}$ of mixed anesthetics per $20 \mathrm{~g}$ body weight, which would prolong the anesthesia for at least $30 \mathrm{~min}$.

\subsection{Enhancement of dental cement adhesion}

The dry and coarse skull surface is helpful for adhesion of dental cement. Application of absolute ethyl alcohol on the skull surface by cotton swab can dehydrate the skull, which facilitates the cement adhesion after ethanol volatilization. A tiny screw can also be useful for fastening of cement on the rest skull region. Before tightening the screws, a drilled shallow hole can make the operation simple, but avoid penetrating the skull.

\subsection{Fiber photometry of axon terminal calcium dynamics}

Due to the overlapping of excitation wavelength $(473 \mathrm{~nm})$ of GCaMP6s with ChR2, the optogenetical stimulation interferes with calcium recording in the same brain region, and vice versa. However, during calcium recording of the axon terminals of V1 neurons, we can rule out the interference between two operations as described in the following. Firstly, the distance between the optogenetical stimulating site (soma body of V1 neuron) and the recording site (axon terminals in LP) is $2.63 \mathrm{~mm}$, which reduced the possibility of interference between them. Secondly, the excitation of recording fiber was adjusted at low intensity of $100 \mu \mathrm{W}$, which will not activate V1 neuron soma. Finally, the insertion of recording fiber is deeper than stimulation fiber, so the soma of V1 neuron would not be affected by excitation of recording fiber.

\subsection{Equipment calibration}

Optogenetics laser intensity was measured by photometer at $473 \mathrm{~nm}$, and the output capacity is fixed $(700 \mu \mathrm{W}$ output at $100 \mathrm{~ms}$ duration and $5 \%$ duty ratio). Recorded output of fiber photometry was analyzed by in DSView software. Background grid 
was adjusted to $2 \mathrm{v} /$ div. and $10 \mathrm{~s} /$ div., which makes the wave type cliffy and scouting easily. Through adjusting laser output intensity, base line can be adjusted to reference line at $1.50 \mathrm{~V}$, thus neural activity recording will not exceed the maximum detection threshold. If GCaMP6s expression level is high, the signal amplifier for PMT should be turned lower at $10^{5}$ times to avoid recorded value out of detection threshold. Similarly, the amplifier should be adjusted to high level $\left(10^{7}\right.$ times) when GCaMP6s expression is low.

\subsection{Postoperative care of surgical mice}

The surgical mice need to be cared in incubator at $37^{\circ} \mathrm{C}$ for $6 \mathrm{~h}$ and feed on jelly food.

\subsection{Modification of virus-based expression system}

Many studies in neuroscience need to be carried out on multi-sites, real-time, awake animal, special structure (synapse, soma body, spine and cytoplasmic organoids) and specific input or output to target neurons. To realize these demands, we can modify the virus-based expression system by constructing the viral promoter and fusing the specific subcellular localization signal peptide sequence with inserted proteins gene such as GECIs, optogenetics proteins,${ }^{15}$ designer receptors exclusively activated by designer drugs (DREADDs) ${ }^{16}$ etc.

\subsection{Application of in vivo fiber photometry}

In vivo fiber photometry is a powerful technique to detect the real-time information of animal brain due to minimal invasion, deep brain recording, easy manipulation and low cost. For two photon microscope, it is hard to detect neural activity in freely moving animals which is also limited in deep brain imaging. Along the promotion of fluorescent indicator for neurotransmitter, potential, ions and so on, the fiber photometry can also be used for detecting the change of glutamine release, potential reactive oxygen species, zinc ion, mitochondrial membrane potential and nitric oxide, etc. ${ }^{17-28}$ Meanwhile, in vivo fiber detection system is convenient for long term and frequent recording during aging, development, learning and memory, and behavioral study which might last for long duration.
However, fiber photometry still cannot reach the single cell resolution of in vivo animal.

\section{Conclusions}

We described a detailed protocol of fiber photometry in freely moving mouse, including virus injection, fiber stub embedding as well as brain $\mathrm{Ca}^{2+}$ signal recording under optogenetical stimulation. This protocol would be helpful for the neuroscience researchers to carry out in vivo functional study through detecting population neural activity by fiber photometry. Together with the advance of optogenetics, genetic sensor, $\mathrm{Ca}^{2+}$ sensor development and viral-based expressing system, this technique will greatly contribute to dissect and elucidate the function of neural circuits.

\section{Acknowledgments}

We thank Convergence Technology Company (\#1037 Luoyu Road, Wuhan, China, website: http://www.cvgc.cn/) for technical support and kind help. This work was supported by the National Natural Science Foundation of China (Grant Nos. 31371106 and 91632110) and HZAU Independent Innovation Fund (2014BQ019).

\section{References}

1. C. K. Kim, S. J. Yang, N. Pichamoorthy, N. P. Young, I. Kauvar, J. H. Jennings, T. N. Lerner, A. Berndt, S. Y. Lee, C. Ramakrishnan, T. J. Davidson, M. Inoue, H. Bito, K. Deisseroth, "Simultaneous fast measurement of circuit dynamics at multiple sites across the mammalian brain," Nat. Methods 13, 325-328 (2016) doi: 10.1038/ nmeth.3770.

2. Q. Guo, J. Zhou, Q. Feng, R. Lin, H. Gong, Q. Luo, S. Zeng, M. Luo, L. Fu, "Multi-channel fiber photometry for population neuronal activity recording," Biomed. Opt. Express 6, 3919-3931 (2015) doi: 10.1364/BOE.6.003919.

3. J. T. Russell, "Imaging calcium signals in vivo: A powerful tool in physiology and pharmacology," $\mathrm{Br}$. J. Pharmacol. 163, 1605-1625 (2011) doi: 10.1111/ j.1476-5381.2010.00988.x.

4. C. Grienberger, A. Konnerth, "Imaging calcium in neurons," Neuron 73, 862-885 (2012) doi: 10.1016/ j.neuron.2012.02.011.

5. J. M. Gee, M. B. Gibbons, M. Taheri, S. Palumbos, S. C. Morris, R. M. Smeal, K. F. Flynn, 
M. N. Economo, C. G. Cizek, M. R. Capecchi, P. Tvrdik, K. S. Wilcox, J. A. White, "Imaging activity in astrocytes and neurons with genetically encoded calcium indicators following in utero electroporation," Front. Mol. Neurosci. 8, 10 (2015) doi: 10.3389/fnmol.2015.00010.

6. J. Akerboom, T. W. Chen, T. J. Wardill, L. Tian, J. S. Marvin, S. Mutlu, N. C. Calderon, F. Esposti, B. G. Borghuis, X. R. Sun, A. Gordus, M. B. Orger, R. Portugues, F. Engert, J. J. Macklin, A. Filosa, A. Aggarwal, R. A. Kerr, R. Takagi, S. Kracun, E. Shigetomi, B. S. Khakh, H. Baier, L. Lagnado, S. S. Wang, C. I. Bargmann, B. E. Kimmel, V. Jayaraman, K. Svoboda, D. S. Kim, E. R. Schreiter, L. L. Looger, "Optimization of a GCaMP calcium indicator for neural activity imaging," J. Neurosci. 32, 13819-13840 (2012) doi: 10.1523/JNEUROSCI.2601-12.2012.

7. Y. Zhao, S. Araki, J. Wu, T. Teramoto, Y. F. Chang, M. Nakano, A. S. Abdelfattah, M. Fujiwara, T. Ishihara, T. Nagai and R. E. Campbell, "An expanded palette of genetically encoded $\mathrm{Ca}(2)(+)$ indicators," Science 333, 1888-1891 (2011) doi: 10.1126/science.1208592.

8. M. I. Kotlikoff, "Genetically encoded $\mathrm{Ca} 2+$ indicators: Using genetics and molecular design to understand complex physiology," J. Physiol. 578, 55-67 (2007) doi: 10.1113/jphysiol.2006.120212.

9. E. D. Papadakis, S. A. Nicklin, A. H. Baker, S. J. White, "Promoters and control elements: Designing expression cassettes for gene therapy," Curr. Gene. Ther. 4, 89-113 (2004).

10. A. Cetin, S. Komai, M. Eliava, P. H. Seeburg, P. Osten, "Stereotaxic gene delivery in the rodent brain," Nat. Protoc. 1, 3166-3173 (2006) doi: 10.1038/nprot.2006.450.

11. B. Mathon, M. Nassar, J. Simonnet, C. Le Duigou, S. Clemenceau, R. Miles, D. Fricker, "Increasing the effectiveness of intracerebral injections in adult and neonatal mice: A neurosurgical point of view," Neurosci. Bull. 31, 685-696 (2015) doi: 10.1007/ s12264-015-1558-0.

12. T. W. Chen, T. J. Wardill, Y. Sun, S. R. Pulver, S. L. Renninger, A. Baohan, E. R. Schreiter, R. A. Kerr, M. B. Orger, V. Jayaraman, L. L. Looger, K. Svoboda, D. S. Kim, "Ultrasensitive fluorescent proteins for imaging neuronal activity," Nature 499, 295-300 (2013) doi: 10.1038/nature12354.

13. K. S. Girven, D. R. Sparta, "Probing deep brain circuitry: New advances in in vivo calcium measurement strategies," ACS Chem. Neurosci. (2016) doi: 10.1021/acschemneuro.6b00307.

14. L. A. Gunaydin, L. Grosenick, J. C. Finkelstein, I. V. Kauvar, L. E. Fenno, A. Adhikari, S. Lammel, J. J. Mirzabekov, R. D. Airan, K. A. Zalocusky,
K. M. Tye, P. Anikeeva, R. C. Malenka, K. Deisseroth, "Natural neural projection dynamics underlying social behavior," Cell 157, 1535-1551 (2014) doi: 10.1016/j.cell.2014.05.017.

15. T. Shimano, B. Fyk-Kolodziej, N. Mirza, M. Asako, K. Tomoda, S. Bledsoe, Z. H. Pan, S. Molitor, A. G. Holt, "Assessment of the AAV-mediated expression of channelrhodopsin-2 and halorhodopsin in brainstem neurons mediating auditory signaling," Brain. Res. 1511, 138-152 (2013) doi: 10.1016/j.brainres. 2012.10.030.

16. M. J. Krashes, S. Koda, C. Ye, S. C. Rogan, A. C. Adams, D. S. Cusher, E. Maratos-Flier, B. L. Roth, B. B. Lowell, "Rapid, reversible activation of AgRP neurons drives feeding behavior in mice," J. Clin. Invest. 121, 1424-1428 (2011) doi: 10.1172/ JCI46229.

17. N. Zhao, S. Chen, Y. Hong and B. Z. Tang, "A red emitting mitochondria-targeted AIE probe as an indicator for membrane potential and mouse sperm activity," Chem. Commun. (Camb.) 51, 1359913602 (2015) doi: 10.1039/c5cc04731e.

18. Z. Han, L. Jin, F. Chen, J. J. Loturco, L. B. Cohen, A. Bondar, J. Lazar and V. A. Pieribone, "Mechanistic studies of the genetically encoded fluorescent protein voltage probe ArcLight," PLoS One 9, e113873 (2014) doi: 10.1371/journal. pone.0113873.

19. J. S. Marvin, B. G. Borghuis, L. Tian, J. Cichon, M. T. Harnett, J. Akerboom, A. Gordus, S. L. Renninger, T. W. Chen, C. I. Bargmann, M. B. Orger, E. R. Schreiter, J. B. Demb, W. B. Gan, S. A. Hires, L. L. Looger, "An optimized fluorescent probe for visualizing glutamate neurotransmission," Nat. Methods. 10, 162-170 (2013) doi: 10.1038/ nmeth.2333.

20. R. S. Gibson, "A historical review of progress in the assessment of dietary zinc intake as an indicator of population zinc status," Adv. Nutr. 3, 772-782 (2012) doi: 10.3945/an.112.002287.

21. L. Barnett, J. Platisa, M. Popovic, V. A. Pieribone, T. Hughes, "A fluorescent, genetically-encoded voltage probe capable of resolving action potentials," PLoS One 7, e43454 (2012) doi: 10.1371/journal.pone.0043454.

22. K. Sreenath, J. R. Allen, M. W. Davidson, L. A. Zhu, "FRET-based indicator for imaging mitochondrial zinc ions," Chem. Commun. (Camb.) 47, 11730-11732 (2011) doi: 10.1039/c1cc14580k.

23. D. Li, S. Chen, E. A. Bellomo, A. I. Tarasov, C. Kaut, G. A. Rutter, W. H. Li, "Imaging dynamic insulin release using a fluorescent zinc indicator for monitoring induced exocytotic release (ZIMIR)," Proc. Natl. Acad. Sci. USA 108, 21063-21068 (2011) doi: 10.1073/pnas.1109773109. 
24. W. Wang, H. Fang, L. Groom, A. Cheng, W. Zhang, J. Liu, X. Wang, K. Li, P. Han, M. Zheng, J. Yin, W. Wang, M. P. Mattson, J. P. Kao, E. G. Lakatta, S. S. Sheu, K. Ouyang, J. Chen, R. T. Dirksen, H. Cheng, "Superoxide flashes in single mitochondria," Cell 134, 279-290 (2008) doi: 10.1016/j. cell.2008.06.017.

25. H. Kojima, K. Sakurai, K. Kikuchi, S. Kawahara, Y. Kirino, H. Nagoshi, Y. Hirata, T. Akaike, H. Maeda, T. Nagano, "Development of a fluorescent indicator for the bioimaging of nitric oxide," Biol. Pharm. Bull. 20, 1229-1232 (1997).

26. R. J. McKenzie, G. F. Azzone and T. E. Conover, "Bulk phase proton fluxes during the generation of membrane potential in rat liver mitochondria," $J$. Biol. Chem. 266, 803-809 (1991).

27. R. L. McKenzie, K. P. Gross, "Two-photon excitation of nitric oxide fluorescence as a temperature indicator in unsteady gasdynamic processes," Appl. Opt. 20, 2153-2165 (1981) doi: 10.1364/ AO.20.002153.

28. T. Tomov, "Pyronin-a fluorescent indicator of membrane potential of the mitochondria. Mechanism of action," Acta. Physiol. Pharmacol. Bulg. 4, 62-68 (1978). 